\title{
Genome sequencing and comparative analysis of three Chlamydia pecorum strains associated with different pathogenic outcomes
}

Michelle Sait ${ }^{1,5}$, Morag Livingstone ${ }^{1}$, Ewan M Clark ${ }^{1,6}$, Nick Wheelhouse ${ }^{1}$, Lucy Spalding ${ }^{1}$, Bryan Markey ${ }^{2}$, Simone Magnino ${ }^{3}$, Frederick A Lainson ${ }^{1}$, Garry SA Myers ${ }^{4}$ and David Longbottom ${ }^{1 *}$

\begin{abstract}
Background: Chlamydia pecorum is the causative agent of a number of acute diseases, but most often causes persistent, subclinical infection in ruminants, swine and birds. In this study, the genome sequences of three $C$. pecorum strains isolated from the faeces of a sheep with inapparent enteric infection (strain W73), from the synovial fluid of a sheep with polyarthritis (strain P787) and from a cervical swab taken from a cow with metritis (strain PV3056/3) were determined using Illumina/Solexa and Roche 454 genome sequencing.

Results: Gene order and synteny was almost identical between C. pecorum strains and C. psittaci. Differences between C. pecorum and other chlamydiae occurred at a number of loci, including the plasticity zone, which contained a MAC/perforin domain protein, two copies of a >3400 amino acid putative cytotoxin gene and four (PV3056/3) or five (P787 and W73) genes encoding phospholipase D. Chlamydia pecorum contains an almost intact tryptophan biosynthesis operon encoding $\operatorname{tr} A B C D F R$ and has the ability to sequester kynurenine from its host, however it lacks the genes folA, folKP and folB required for folate metabolism found in other chlamydiae. A total of 15 polymorphic membrane proteins were identified, belonging to six pmp families. Strains possess an intact type III secretion system composed of 18 structural genes and accessory proteins, however a number of putative inc effector proteins widely distributed in chlamydiae are absent from C. pecorum. Two genes encoding the hypothetical protein ORF663 and IncA contain variable numbers of repeat sequences that could be associated with persistence of infection.
\end{abstract}

Conclusions: Genome sequencing of three C. pecorum strains, originating from animals with different disease manifestations, has identified differences in ORF663 and pseudogene content between strains and has identified genes and metabolic traits that may influence intracellular survival, pathogenicity and evasion of the host immune system.

Keywords: Chlamydia pecorum, Genome sequence, Polymorphic membrane proteins, Plasticity zone, Tryptophan metabolism, Folate biosynthesis, Clustered tandem repeats

\section{Background}

Members of the genus Chlamydia are Gram-negative, obligate intracellular pathogens that share a biphasic developmental cycle. Chlamydia pecorum infects a broad host range, including small and large ruminants, swine, birds and marsupials. Seroprevalence and PCR-based studies suggest that infection or exposure to C. pecorum

\footnotetext{
* Correspondence: david.longbottom@moredun.ac.uk

${ }^{1}$ Moredun Research Institute, Pentlands Science Park, Bush Loan, Edinburgh, Midlothian EH26 OPZ, UK

Full list of author information is available at the end of the article
}

and/or C. abortus is almost ubiquitous in cattle and sheep [1-5]. In the majority of these cases, infection is subclinical, with C. pecorum being routinely detected in the intestine and genital tract. The incidence and severity of disease caused by C. pecorum appears to be heightened in koalas and is associated with clinical disease such as conjunctivitis, urinary- and reproductive tract disease, and infertility [6]. Many chlamydial species, including C. pecorum can enter persistent states, characterised in vitro by enlarged, morphologically aberrant, non-fusogenic reticulate bodies (RBs). Persistence can be 
induced in vitro by antibiotic exposure [7], amino acid[8] or iron- [9] deficiencies and exposure to IFN- $\gamma$ [10] and it is likely that $C$. pecorum causes a persistent, subclinical infection in the host. Subclinical infections can have detrimental effects on the animal's health. Animals with inapparent chlamydiae infections have higher body temperatures, lower body weights, reduced growth rates, reduced iron, haemoglobin, haematocrit and leukocyte levels and a higher incidence of follicular bronchiolitis [11-13]. C. pecorum can also cause clinical disease including encephalomyelitis, vaginitis, endometritis, mastitis, conjunctivitis, polyarthritis, pneumonia, enteritis, orchitis, pleuritis, infertility or pericarditis [6].

Genetic variation has been reported to occur between C. pecorum strains in ompA, the $r r n-n q r F$ intergenic region, incA, rRNAs, a number of housekeeping genes and the hypothetical protein ORF663 [14-22]. These and other unidentified genomic differences may enable differentiation between strains isolated from asymptomatic or diseased animals. However, to date, only the genome sequence of a single C. pecorum strain (E58) has been published [23]. The genetic factors responsible for the diverse host range, tissue tropism, disease outcomes and associated sequelae of C. pecorum infections are thus still poorly understood. In this study, we present the complete genome sequences of three C. pecorum strains isolated from animals exhibiting different disease manifestations and use comparative genomics to provide insights into the biology of C. pecorum and to identify both genus- and species-specific virulence factors.

\section{Results and discussion}

\section{Genome features and comparative analysis}

The genomes of $C$. pecorum PV3056/3 (CPE1), W73 (CPE2) and P787 (CPE3) each comprise a single circular chromosome of 1,104,552 bp, 1,106,534 bp and 1,106,412 bp, respectively. The general features of these genomes compared to reference strain E58 [GenBank: CP002608] [23] are shown in Figure 1 and Table 1 . The G + C content of each genome is $41.1 \%$ and none of the strains contain any plasmids. The origins of replication were assigned based on base composition asymmetry of the genomes and in each genome the oriC is located upstream of the hem $\mathrm{B}$ gene. There are 38 tRNA genes corresponding to all the amino acids except selenocysteine and pyrrolysine, one rRNA operon, and 3 sRNA molecules corresponding to SsrA, RNaseP and ffs (Additional file 1: Table S1) present in each chromosome. Annotation identified 927 (PV3056/3) and 928 (P787 and W73) predicted coding sequences (CDSs), representing a coding density of $92.5 \%$. Of the predicted CDSs, 628 (67.7\%, PV3056/3), 630 (67.9\%, W73) and 629 (67.8\%, P787) were functionally assigned based on previous experimental evidence or database similarity and motif matches. For hypothetical proteins with no functional assignment, 209 (PV3056/3) and 208 (P787 and W73) proteins (69.8\%) were either unique to $C$. pecorum or significantly similar to proteins from chlamydial species. The number of pseudogenes varied between C. pecorum strains, with the majority occurring due to frameshift mutations in homopolymeric tracts. PV3056/3 contained 6 pseudogenes, while P787 and W73 contained 3 pseudogenes each. Pseudogenes were annotated as phospholipase D family proteins, an ABC transporter protein and hypothetical proteins (Additional file 1: Table S2).

Comparative analysis of the three C. pecorum genomes to reference strain E58 [GenBank: CP002608] [23] revealed a high level of sequence conservation, gene content and order (Figure 2A). Phylogenetic analysis of 48 concatenated ribosomal proteins from Chlamydia species revealed C. pecorum strains to be most closely related to C. pneumoniae (Figure 2B), an observation in agreement with the MLST analysis of several housekeeping genes [24]. However, global comparisons between $C$. pecorum and other chlamydial species reveal gene order and synteny to be most similar to C. psittaci (Figure 2C). Comparisons between C. pecorum P787, C. psittaci 6BC [GenBank: CP002586] and C. pneumoniae AR39 [GenBank: AE002161] show chromosomal rearrangements including a large DNA inversion in the plasticity zone (PZ) of the genome. An additional asymmetrical translocation is observed between C. pecorum and C. pneumoniae in the region flanking the $p m p G$ genes corresponding to the region 322207-381219 in P787 and encoding 55 genes (CPE3_0288-CPE3_0342) (Figure 2C). Comparative analysis between $C$. pecorum and other chlamydial species suggests that genetic rearrangements also occur in the regions flanking the PZ between the conserved orthologs $z w f$, encoding glucose-6-phosphate 1-dehydrogenase (CPE1_0526, CPE2_ 0526, CPE3_0526) and a peptide ABC transporter (CPE1_0575, CPE2_0576, CPE3_0576) spanning a 72.0$73.7 \mathrm{~kb}$ region encoding 46 (PV3056/3) and 47 (W73 and P787) genes (Figure 3).

\section{Metabolic characteristics}

Comparative genomics of chlamydial species has identified a number of genes coding for metabolic functions, such as tryptophan metabolism, biotin biosynthesis and folate biosynthesis, where subtle variations in gene content may contribute to growth of the organism in vivo and the ability to evade the host immune system [23,25-29].

The genome of $C$. pecorum contains an almost intact tryptophan biosynthesis operon, consisting of anthranilate phosphoribosyltransferase $(\operatorname{tr} p D)$, phosphoribosylanthranilate isomerase $(\operatorname{trp} F)$, indole-3-glycerol phosphate synthase $(\operatorname{trp} C)$, tryptophan synthase alpha chain $(\operatorname{trp} A)$ and 


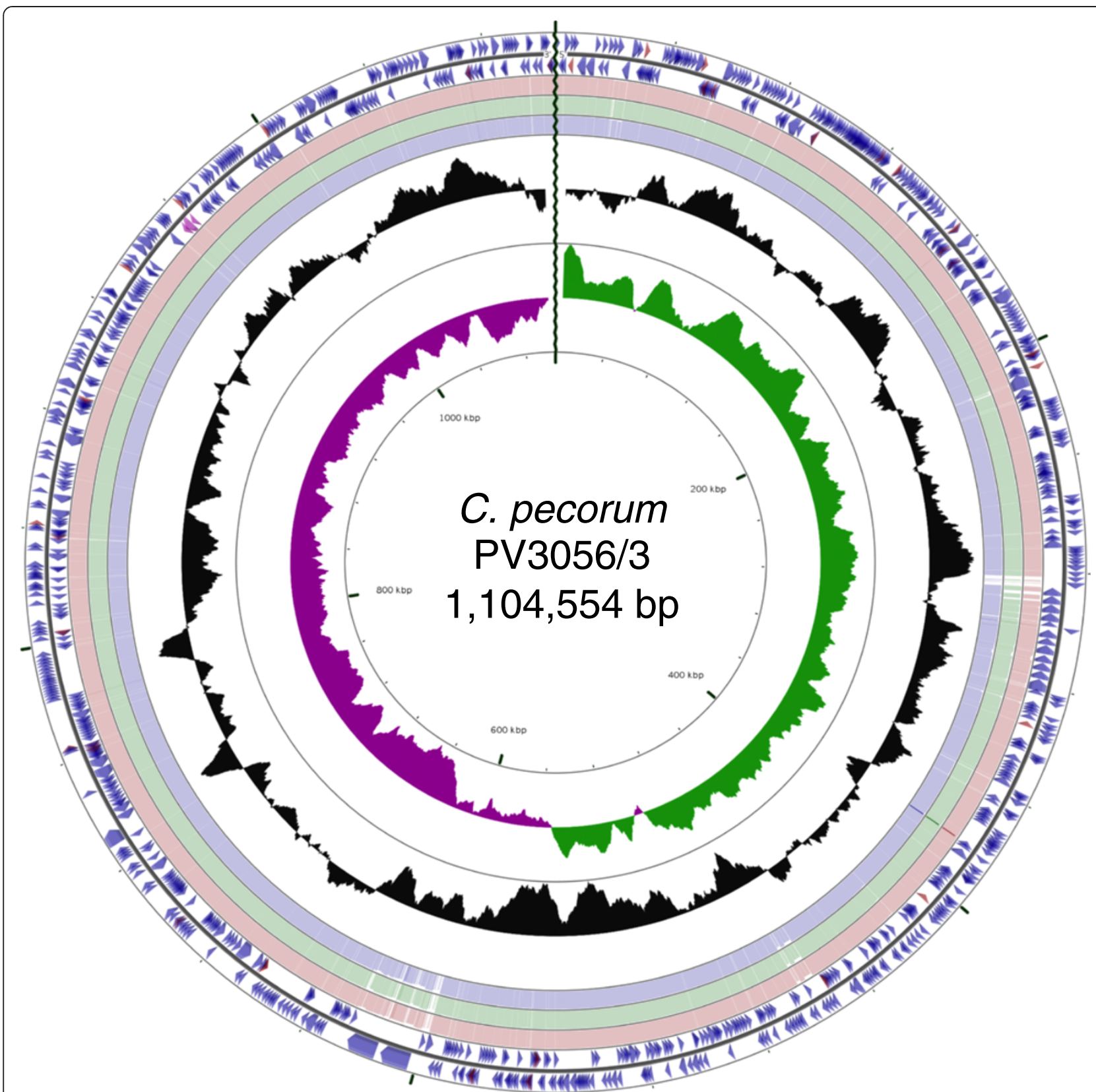

Figure 1 Circular representation of the genome of $C$. pecorum PV3056/3. Circles from the outside in show: the positions of protein-coding genes (blue), tRNA genes (red) and rRNA genes (pink) on the positive (circle 1), and negative (circle 2), strands respectively. Circles 3-5 show the positions of BLAST hits detected through blastn comparisons of PV3056/3 against W73 (circle 3), P787 (circle 4) and E58 (circle 5) with the following settings: query split size $=50,000 \mathrm{bp}$, query split overlap size $=0$, expect value cutoff $=0.00001$. Low complexity sequences were eliminated from the analysis. The height of the shading in the BLAST results rings is proportional to the percent identity of the hit. Overlapping hits appear as darker shading. Circles 6 and 7 show plots of GC content and GC skew plotted as the deviation from the average for the entire sequence. The origin of replication is indicated by the vertical zig-zag line.

tryptophan synthase beta chain $(\operatorname{trp} B)$ genes. This complement of genes and the gene arrangement is most similar to that found in C. caviae, however the tryptophan biosynthesis operon in C. pecorum is not located in the plasticity zone and does not contain the additional $\operatorname{trp} B$ gene found in C. caviae [26]. The complement of genes observed in C. pecorum would theoretically permit the production of tryptophan from the substrate anthranilate. However, the gene complement will not permit the first step of tryptophan biosynthesis, the conversion of chorismate 
Table 1 General features of C. pecorum PV3056/3, P787 and W73 compared with the type strain E58 [CP002608]

\begin{tabular}{lllll}
\hline & C. pecorum (PV3056/3) & C. pecorum (P787) & C. pecorum (W73) & C. pecorum (E58) [23] \\
\hline Date of isolation & 1991 & 1977 & 1989 & Circa 1940 \\
Country & Italy & Scotland & Northern Ireland & USA \\
Source & Cow, cervical swab & Sheep, synovial fluid & Sheep, faeces & Calf, brain \\
Disease pathotype & Metritis & Polyarthritis & Asymptomatic/enteric & Encephylomyelitis \\
Genome size (bp) & $1,104,552$ & $1,106,412$ & $1,106,534$ & $1,106,197$ \\
\% GC of genome & 41.1 & 41.1 & 41.1 & 41.1 \\
\% coding & 91.9 & 92.1 & 92.1 & 91.9 \\
Predicted CDS & 927 & 928 & 928 & 1073 \\
Predicted no. of pseudogenes & 6 & 3 & 3 & 1 \\
No. of CDS with functional assignment & $628(67.7 \%)$ & $629(67.8 \%)$ & $630(67.9 \%)$ & - \\
No. of pmp proteins & 15 & 15 & 15 & 15 \\
No. of tRNA genes & 38 & 38 & 38 & 38 \\
No. of rRNA operons & 1 & 1 & 1 & 1 \\
No. of sRNA molecules & 3 & 3 & 3 & 3 \\
Location of OriC region & $1104229-147 \mathrm{nt}(471 \mathrm{nt})$ & $1106263-147 \mathrm{nt}(296 \mathrm{nt})$ & $1106389-147 \mathrm{nt}(295 \mathrm{nt})$ & $305941-306155 \mathrm{nt}(215 \mathrm{nt})$ \\
\hline
\end{tabular}

to anthranilate, which is catalysed by anthranilate synthetase (trpE/G). The acquisition of anthranilate could be achieved by $C$. pecorum through the direct uptake of kynurenine from the host cell via an aromatic amino acid transporter similar to tyrP (CPE1_0759, CPE2_0760, CPE3_0760), converted to anthranilate by kynureninase (kynU, CPE1_0671, CPE2_0672, CPE3_0672) and further metabolised to phosphoribosyl anthranilate by trpD in the presence of PRPP synthase and then to tryptophan via a series of intermediates (Figure 4). In mammalian cells, the production of the pro-inflammatory cytokine IFN- $\gamma$ by the host has been documented to decrease the availability of L-tryptophan in host cells by the induction of indoleamine 2,3-dioxygenase (IDO) that converts L-tryptophan to Lformylkynurenine and then subsequently to kynurenine by arylformamidase [30]. This limitation of tryptophan by the host can lead either to the resolution of chlamydial infections or the establishment of persistent infections by chlamydial species [31]. The ability of $C$. pecorum to synthesise tryptophan in an IFN- $\gamma$ rich environment may contribute to its ability to form persistent, subclinical infections.

The 3 sequenced $C$. pecorum strains and E58 contain the biotin biosynthesis operon encoding bioBFDA. (CPE1_0687-CPE1_0690; CPE2_0688-CPE2_0691; CP E3_0688-CPE3_0691). This region shows significant variability between chlamydial species, being absent in $C$. caviae, C. trachomatis and C. muridarum but present in C. abortus, C. psittaci, C. felis and C. pneumoniae. The ability to synthesise biotin is hypothesised to assist in the colonization of biotin-limited niches and contribute to the tissue tropism differences observed in the chlamydiae [25]. Upstream of bioBFDA, located between $\operatorname{dap} B$ and bio $B$, a series of genes encoding hypothetical proteins with unknown function and limited distribution across chlamydial species are present. Chlamydia abortus, C. psittaci and C. felis genomes contain four genes (in C. abortus, CAB681, CAB682, CAB683 and CAB684), C. pneumoniae contains 2 genes in this region that are homologues of CAB681 and CAB682, while C. pecorum contains one gene in this region that is homologous to CAB684 (Additional file 2: Figure S1).

Three genes encoding key enzymes involved in folate biosynthesis, namely dihydrofolate reductase ( $f o l A)$, dihydropteroate synthase $(f o l K P)$ and dihydroneopterin aldolase $(f o l B)$, are absent from all 4 C. pecorum genomes (Figure 5). These genes are present in other chlamydiaceae species (C. abortus, C. psittaci, C. caviae, C. felis, C. pneumoniae, C. muridarum and C. trachomatis) (Figure 5, Additional file 1: Table S3). These findings suggest that C. pecorum will be unable to synthesize folate or 7,8dihydrofolate (DHF) and may require an exogenous source. In members of the Firmicutes, this is achieved through active transport systems [32], however homologues to these could not be identified in C. pecorum. The absence of genes folA and folKP in C. pecorum would theoretically confer a natural resistance to trimethoprim and sulphonamide antibiotics, which act as substrate analogues of dihydrofolate reductase (FolA) and dihydropteroate synthase (FolKP), respectively. The 


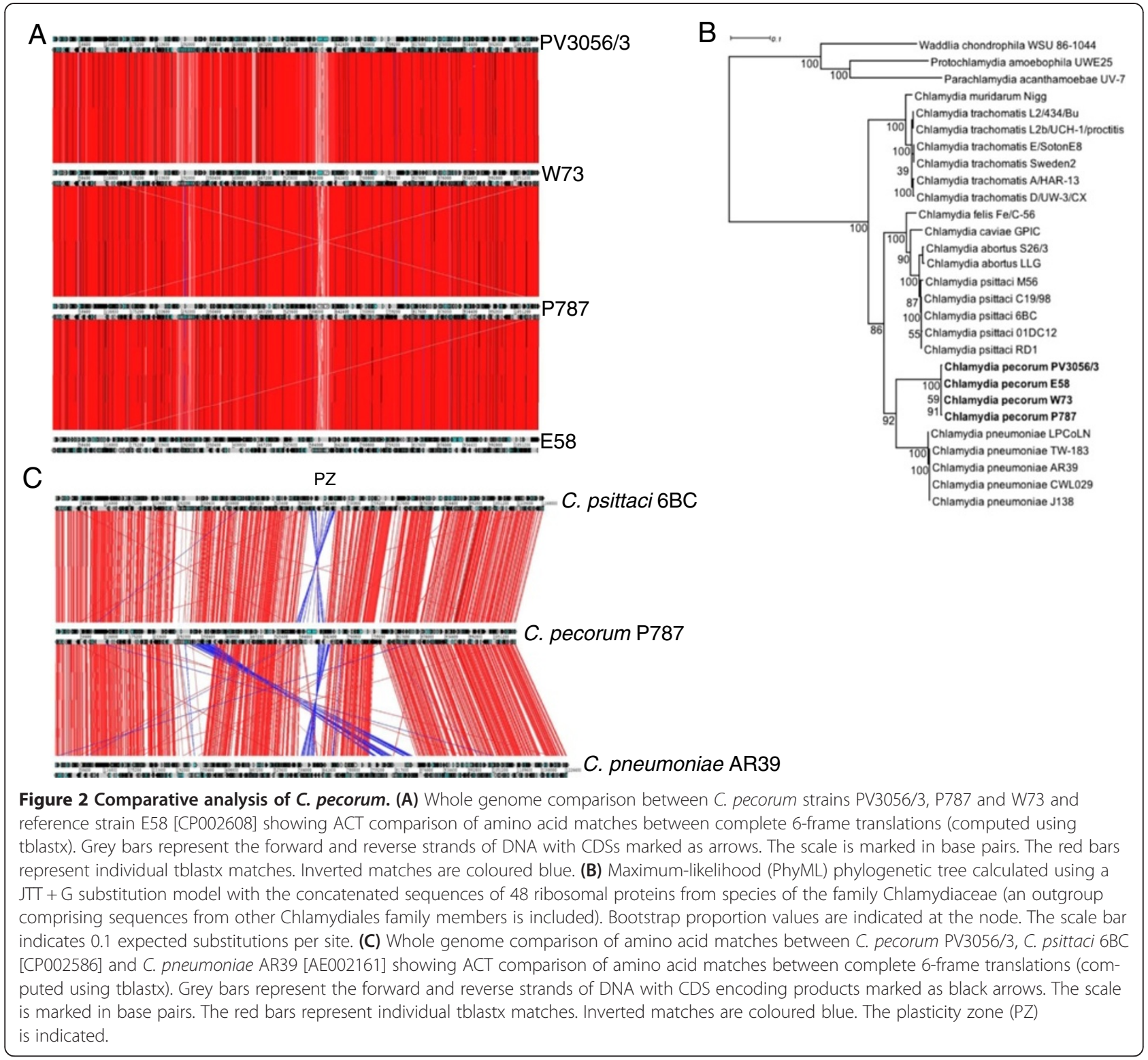

absence of genes thy $A$ (classical thymidylate synthase) and folA in all C. pecorum genomes indicates that the formation of 5,6,7,8-tetrahydrofolate (THF), an essential donor of one-carbon units for DNA, RNA and protein syntheses, must be achieved through other pathways. Indeed, all Chlamydiaceae species sequenced to date, including C. pecorum, contain homologs for thyX (also known as thy1), glyA, folD, $y g f A$ and $f m t$ that encode enzymes allowing the synthesis and interconversion of carbon-one folate derivatives (Figure 5, Additional file 1: Table S3) in the production of dTMP (thymidylate; required for DNA synthesis) and formylmethionine (initiator methionine for protein synthesis). The flavin-dependent alternative thymidylate synthase ThyX uses 5,10-methylenetetrahydrofolate as a one-carbon donor and links dTMP catalysis with the formation of THF [33]. However, bacteria with a thy $X^{+} /$fol $A^{-} /$thy $A^{-}$genotype, like $C$. pecorum, must still contain reduced folates for RNA and protein synthesis to take place. This is likely achieved through an alternate pathway involving other enzymes encoded by $g l y A$ (serine hydroxymethyltransferase), folD (methylene tetrahydrofolate cyclohydrolase/dehydrogenase), $y g f A$ (5-formyltetrahydrofolate cyclo-ligase) and fmt (methionyl-tRNA formyltransferase). The novel folate cycle observed in $C$. 


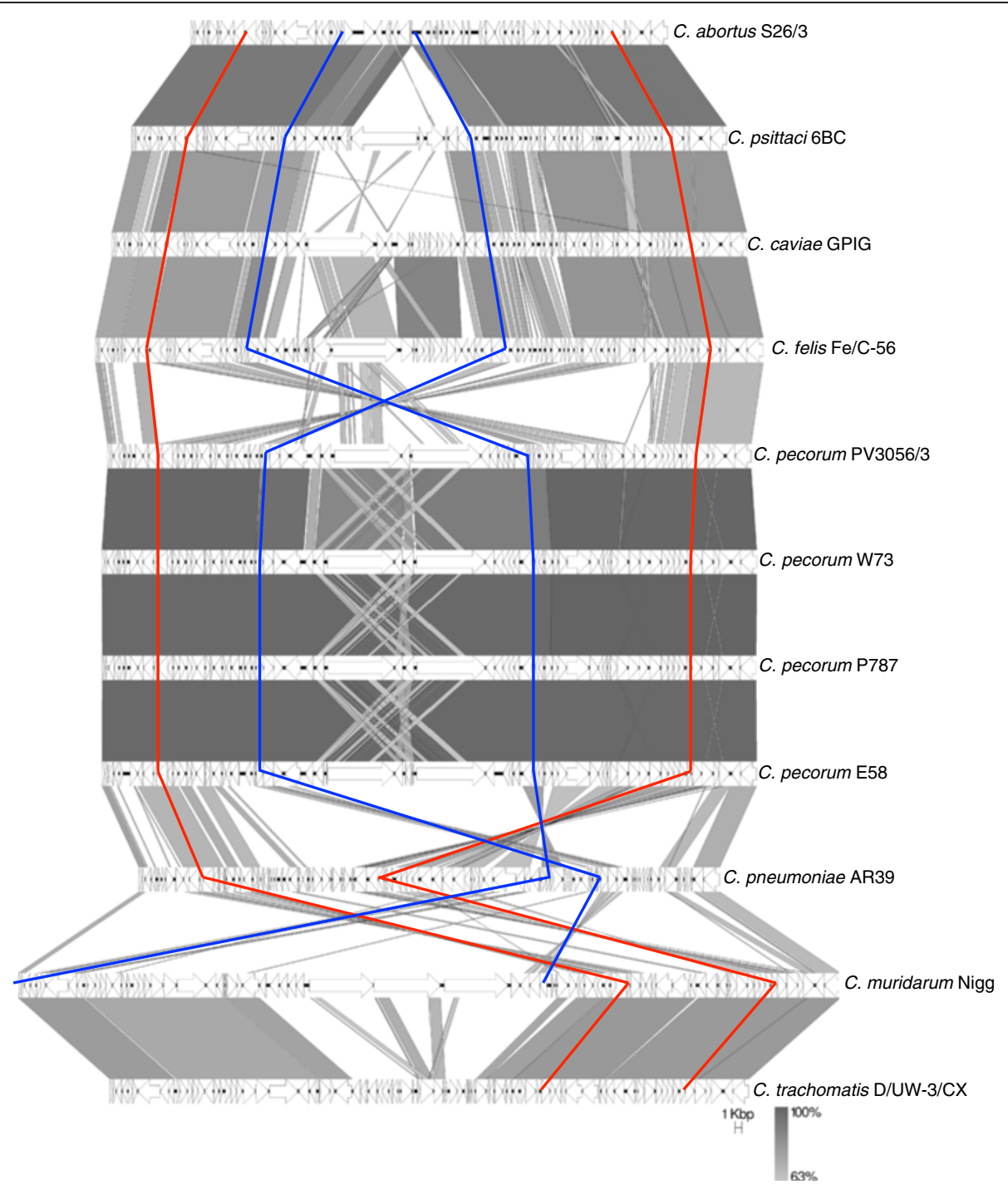

Figure 3 Comparison of genomic regions flanking the C. pecorum plasticity zone. Comparison of nucleotide matches (computed using blastn) between the genes encoding glucose-6-phosphate 1-dehydrogenase and a peptide ABC transporter (indicated by red vertical lines) for available representative genomes of the family Chlamydiaceae. CDSs are marked as arrows. The blue vertical line represents the plasticity zone defined as the regions between $a c c B$ and guaB. The depth of shading is indicative of the percentage blastn match, as indicated bottom right.

The scale is marked in kilobase pairs.

pecorum may contribute to the occurrence of persistent infections due to the limited pool of reduced folates available to the cell. As C. pecorum is likely to acquire folate directly from the host cell, increased competition could result in folate deficiency in the host, contributing to the increased levels of anaemia and lower body weights observed in infected animals $[11,13]$.

\section{Bacterial secretion systems}

The C. pecorum genomes each contain 15 genes that encode members of the type $\mathrm{V}$ "autotransporter (AT)" secretion system (Figure 6A). In chlamydial species, these are referred to as polymorphic membrane proteins (pmps) and are present in all sequenced genomes, in numbers ranging from 9 in C. trachomatis to 21 in C. pneumoniae. C. pecorum ATs range in predicted size and $\mathrm{pI}$ from 89 to $176 \mathrm{kDa}$ and 5.05 to 8.93 respectively (Additional file 1: Table S4) and possess the conserved AT domain architecture comprising a central pmpM domain, C-terminal autotransporter (AT) domain and predicted passengerdomains with a variable number of the repeat motifs GG $[\mathrm{A} / \mathrm{L} / \mathrm{V} / \mathrm{I}][\mathrm{I} / \mathrm{L} / \mathrm{V} / \mathrm{Y}]$ and $\mathrm{FXXN}$ [34]. N-terminal signal sequences with potential signal peptidase 1 cleavage sites were identified in 12 ATs (Figure 6B, Additional file 1: Table S4). Phylogenetic analysis of the C-terminal AT domains identified the C. pecorum ATs as belonging to 6 gene families. Individual gene families showed high 


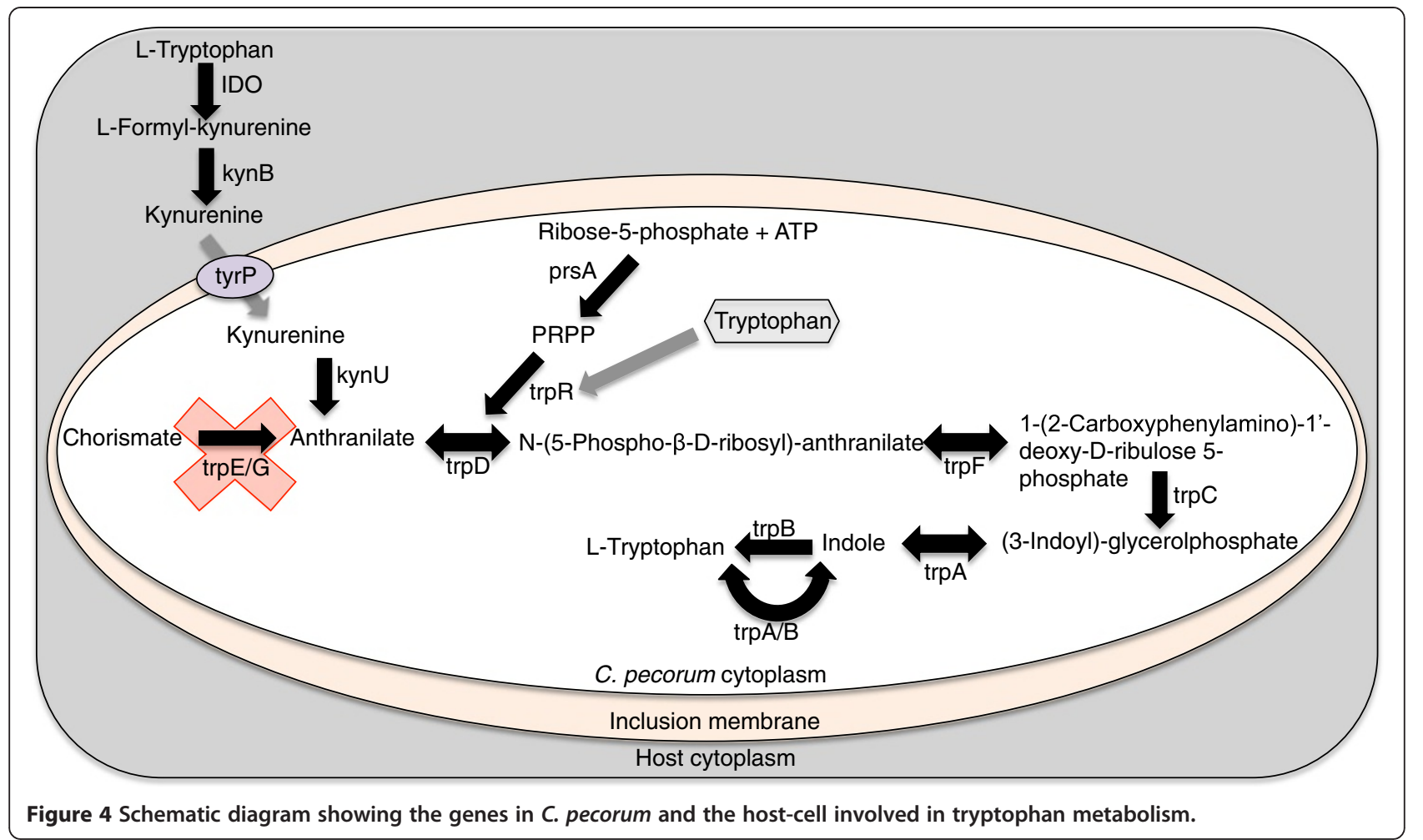

bootstrap support (>97\%) but only weak support at deeper branches (35-87\%) (Figure 6C). Phylogenetic network analysis performed on AT sequences show separation into the AT gene families but suggests that recombination is occurring between AT domains (phi test for recombination $\mathrm{p}=0.02173$ ) (Additional file 2: Figure S2). ATs were located in 4 genetic loci consisting of two singletons belonging to the pmpD (CPE1_0766, CPE2_0767, CPE3_0767) and pmpG (CPE1_0679, CPE2_0680, CPE3_0680) protein families, two pairs of genes belonging to $\mathrm{pmpB}$ and $\mathrm{pmpA}$ (CPE1_0210, CPE2_0210, CPE3_0210; CPE1_0211, CPE2_0 211, CPE3_0211), and a cluster of 11 genes belonging to the pmpE (CPE1_0275-0276, CPE2_0275-0276, CPE3_02 75-0276), pmpH (CPE1_0277, CPE2_0277, CPE3_0277) and pmpG (CPE1_0278, CPE1_0281-0287, CPE2_0278, CPE2_0281-0287, CPE3_0278, CPE3_0281-0287) protein families (Figure 6A, 6C, Additional file 2: Figure S2). All AT-encoding genes were intact in $C$. pecorum except for the gene encoding pmpA in E58 (G5S_0527). Based on the short length of homopolymeric tracts identified in C. pecorum ATs (maximum 8 nucleotides), it appears less likely that expression of these genes are subject to phase variation by strand slippage mechanisms compared to ATs from other organisms such as C. abortus (maximum 16 nucleotides).

The Type III secretion system (T3SS) consists of 18 genes encoding the major structural components of the secretion apparatus, accessory proteins and chaperones and is arranged in 4 genetic loci (Additional file 1: Table S5). In sequenced chlamydial genomes, a number of putative T3SS effector proteins belonging to the Inc or transmembrane head $(\mathrm{TMH})$ protein family are located in the region extending between pmpD and $1 \mathrm{pxB}$ (Additional file 2: Figure S3). The distance between the 3 ' ends of these genes in C. pecorum is $\sim 2.8 \mathrm{~kb}$ (2 genes) compared to $18.1 \mathrm{~kb}$ C. abortus (11 genes), $17.7 \mathrm{~kb}$ in C. psittaci (11 genes), $16.4 \mathrm{~kb}$ in C. caviae (13 genes), $15.9 \mathrm{~kb}$ in C. felis (11 genes) and $1.7 \mathrm{~kb}$ in C. pneumoniae (1 gene). The two genes present in this region in C. pecorum (CPE1_0764 (pseudogene), CPE1_0765, CPE2_0765, CPE2_0766, CPE 3_0765, CPE3_0766) possess an N-terminal signal sequence, a single $\mathrm{N}$-terminal transmembrane domain and two domains of unknown function (DUF1539 and DUF1548). Members of this protein family are present in C. abortus, C. psittaci, C. caviae and C. felis (3 CDSs each) and C. pneumoniae (1 CDS).

\section{Simple sequence repeats}

A region of variability between $C$. pecorum and other chlamydial species is located immediately upstream of the $5 \mathrm{~S}$ rRNA gene. This region, between the 3 ' ends of the $5 \mathrm{~S}$ rRNA and $n q r F$ genes range in size from 261-269 bp in $C$. pecorum to $4464 \mathrm{bp}$ in $C$. caviae. In $C$. caviae this region encodes a 1291aa residue pseudogene identified as a member of the virulence-associated invasion/intimin family of outer membrane proteins of Gram-negative bacteria. The genome of C. abortus contains two CDSs in place of the intimin family gene in this region, 


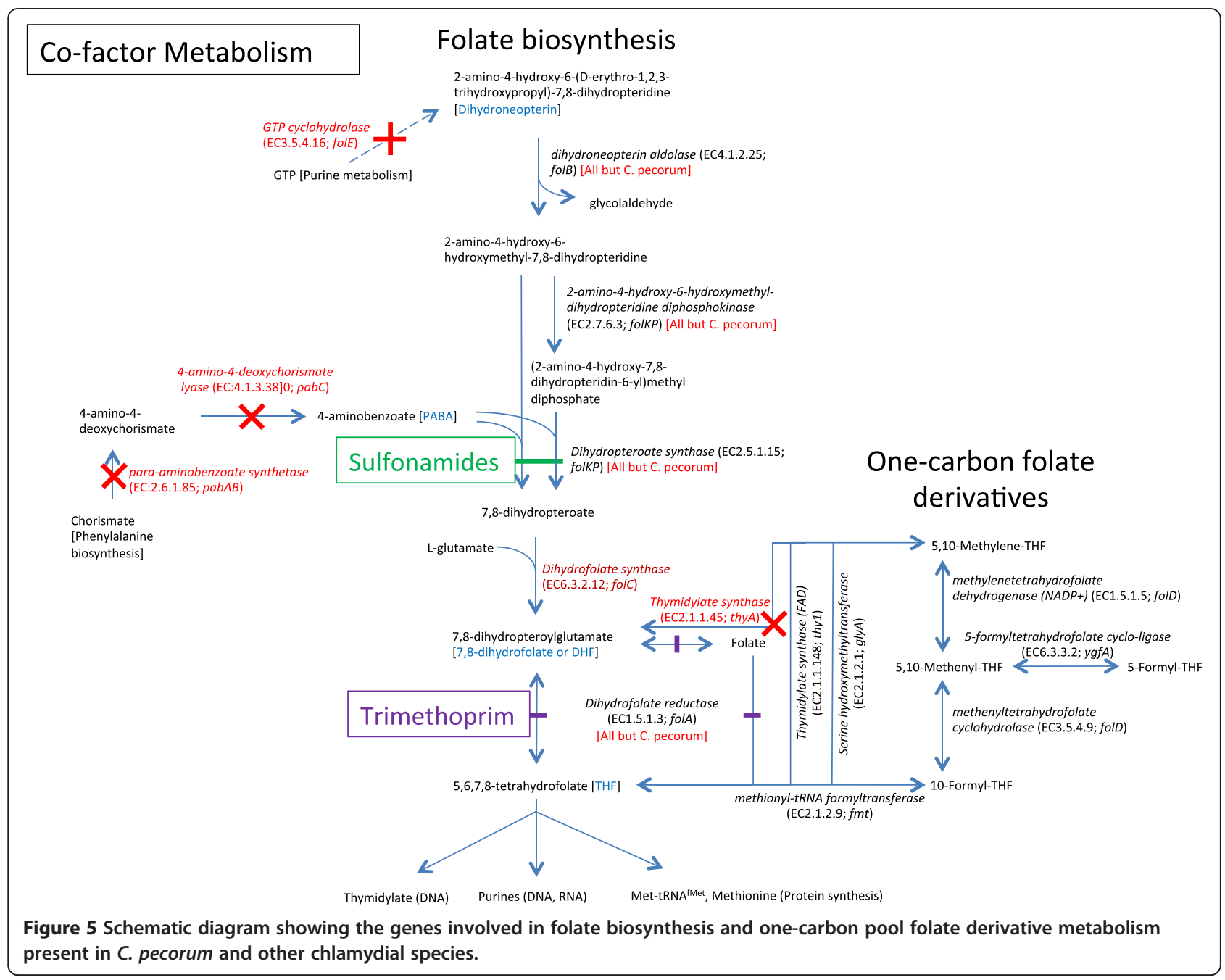

encoding a conserved membrane protein and a unique hypothetical protein. In C. psittaci, C. felis and C. muridarum these two proteins are fused to encode a single hypothetical protein. In C. pecorum there are no predicted CDSs in this region and the intergenic region between the $5 \mathrm{~S}$ rRNA and $n q r F$ genes comprises an 8 bp simple sequence repeat sequence AAAGCACT repeated 12 (W73, PV3056/3 and E58) or 13 times (P787) (Additional file 2: Figure S4).

Clustered tandem repeat sequences (CTRs) appearing in the hypothetical protein ORF663 (CPE1_0343, CPE2_0343, CPE3_0343) have been used to differentiate between pathogenic and non-pathogenic strains of $C$. pecorum with non-pathogenic strains containing a greater number of CTRs [21]. The C. pecorum strains contained different numbers and types of CTRs varying from 14-27 CTRs in the C. pecorum strains originating from diseased animals (PV3056/3 and P787) to 52 CTRs in W73 that was isolated from an animal with subclinical disease (Table 2). Whilst no predicted function has been assigned to ORF663, N-terminal signal peptides and two transmembrane domains were identified in the corresponding genes of PV3056/3, W73 and P787 suggesting that the protein may be surface expressed. Indeed, the high proportions of serine (13.3-18.0\%), proline (10.7-14.9\%) and lysine (10.7-14.6\%) in ORF663 could indicate adhesion functions, such as those observed in Staphylococcus sp. and Streptococcus sp. [35,36]. In Streptococcus sp. correlations between the number of CTRs and pathogenicity has been reported, with deletions in the CTR causing either a loss of conformational epitopes or a decrease in the antigen size and reduction in antibody binding to the bacterial surface, resulting in increased pathogenicity [37] and it is feasible that this also occurs in chlamydiae.

The IncA protein is an effector protein secreted by the type III secretion system (T3SS) that is known to localize to the chlamydial inclusion membrane [38]. In $C$. pecorum, IncA has been identified as an antigen that can be used for serodiagnosis [39], and the identification and survey of CTR sequences in incA from isolates 

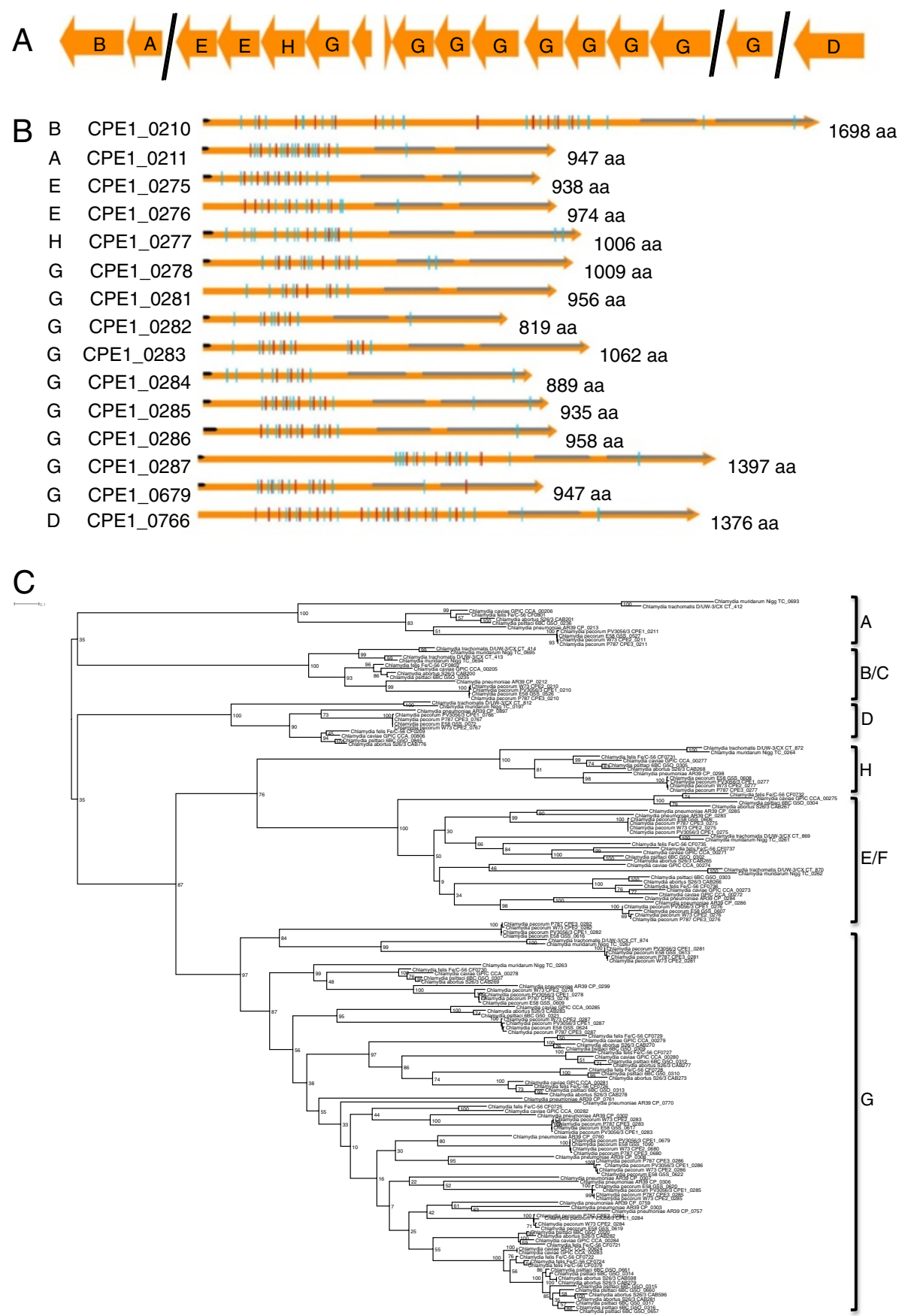

Figure 6 Polymorphic membrane proteins in C. pecorum. (A) Genetic organisation of Pmps in C. pecorum with gene families (as indicated) identified following BLAST and phylogenetic comparison (see C. below) with other published Pmps. (B) Schematic diagram showing the conserved Pmp features, comprising: predicted pmpM and autotransporter domains (grey arrows); predicted pmp passenger domain repeat motifs GG[A/LN/I][/LN/Y] (blue vertical lines); and FXXN motifs (red vertical lines). Signal peptide sequences are as indicated (black arrows). The predicted number of amino acids (aa) is indicated to the right of the gene. Gene families (see $\mathbf{C}$. below) are indicated to the left of the locus tags. (C) Maximum-likelihood (PhyML) phylogenetic tree of autotransporter domains showing clustering of Pmps into gene families (indicated to the right of the groups). For clarity the figure shown displays a subset of 121 sequences based on a larger alignment of 367 sequences. Trees were calculated using a JTT $+1+G$ substitution model. Bootstrap support is indicated by number at the node. The scale bar indicates 0.1 expected substitutions per site. 
Table 2 Clustered tandem repeat (CTR) sequences observed in orthologs of hypothetical protein ORF663

\begin{tabular}{|c|c|c|c|c|c|c|c|c|c|c|c|c|c|c|c|c|}
\hline \multirow[b]{2}{*}{ Strain } & \multicolumn{15}{|c|}{ CTR motif sequence } & \multirow[b]{2}{*}{ Total } \\
\hline & KEPST & KEPSK & KELSP & KEPLP & KESSP & KKPSP & KEPSS & KEPSK & KSLHL & KNLHL & KNFHL & KNSHL & KNLYL & KNLQS & KEPSP & \\
\hline PV3056/3 & 26 & 1 & & & & & & & & & & & & & & 27 \\
\hline W73 & & & 27 & 2 & 15 & 6 & 1 & 1 & & & & & & & & 52 \\
\hline P787 & & & 1 & 2 & & & 1 & & 2 & 3 & 1 & 2 & 1 & 1 & & 14 \\
\hline E58 & & & 7 & & & & 6 & 1 & & & & & & & 8 & 22 \\
\hline
\end{tabular}

originating from symptomatic and asymptomatic animals suggest that the incA CTR motif composition in $C$. pecorum could be associated with virulence [21]. The number and composition of incA CTRs in the sequenced genomes varied from 8 in P787 to 12 in W73 (Table 3$)$. This differs from those previously reported for E58 $(12 \times$ APA $)$ and W73 $(2 \times$ APA and $8 \times$ APAPE $)$ [21]. The differences observed in these CTRs between strains held in different laboratories could result from adaptation of the strains to laboratory growth conditions. As IncA has been shown to contribute in establishing interactions between the inclusion and the host cell, participating in vesicle fusion or septation of the inclusion membrane during bacterial cell division [40], the presence of CTRs could contribute to the ability of C. pecorum to evade the host immune system or contribute to the formation of sub-clinical infections by forming non-fusogenic inclusions $[21,41]$.

\section{Plasticity zone}

In chlamydial species, the plasticity zone is defined as the region between inosine-5' -monophosphate dehydrogenase $(g u a B)$ and acetyl-CoA carboxylase $(a c c B)$ and is the region of the genome that is most variable in gene content and sequence. In C. pecorum, this region is 40.3-42.1 kb in size and contains 16 (PV3056/3) or 17 (W73 and P787) genes encoding GMP synthase, an adenosine deaminase superfamily-protein, a MAC/perforin domain-containing protein, 3 (PV3056/3) or 4 (W73 and P787) phospholipase D family proteins, 2 cytotoxins and 4 hypothetical proteins (Additional file 1: Table S6).

The presence of two cytotoxin genes in the PZ of each of the sequenced C. pecorum strains (CPE1_0552, CPE 1_0554, CPE2_0552, CPE2_0555, CPE3_0552, CPE3_0555) may contribute to the ability of the organism to switch

Table 3 Clustered tandem repeat sequences (CTR) observed in IncA

\begin{tabular}{llllll}
\hline & \multicolumn{3}{c}{ CTR motif sequence } & \\
\cline { 2 - 4 } Strain & APA & APAE & APE & AP & Total \\
\hline PV3056/3 & 9 & & 1 & & 10 \\
W73 & 1 & 11 & & 1 & 12 \\
P787 & 2 & 5 & & 1 & 8 \\
E58 & 11 & 1 & & & 12 \\
\hline
\end{tabular}

from persistent infection to causing acute disease. The cytotoxin genes share sequence similarity with $E$. coli and Citrobacter rodentium lymphocyte inhibitory factor A (lifA) and Clostridium difficile toxin B as well as other chlamydial cytotoxins. The 10-10.3 kb cytotoxins in C. pecorum consist of an $\mathrm{N}$-terminal glucosyltransferase domain responsible for the biological effects of the toxin, a cysteine protease domain responsible for autocatalytic cleavage and a large domain of unknown function that may play a role in cytotoxin translocation or receptor binding. Phylogenetic analysis of cytotoxins from C. psittaci, C. felis and C. cavie (1 copy each), C. pecorum (2 copies each) and C. muridarum (3 copies) reveals extensive diversity within these genes (Figure 7). C. pecorum cytotoxins belonged to two separate gene clusters (Cluster 1:CPE1_0552, CPE2_0552, CPE3_0552; Cluster 2:CPE1_0554, CPE2_0555, CPE3_ 0555) each showing greatest similarity to cytotoxins from C. muridarum. It is unclear whether the two different cytotoxins in $C$. pecorum have different biological functions or host specificity. Related cytotoxins in E. coli and C. difficile act by glycosylating small GTP-binding proteins of Rho and Ras families, inhibiting the host signalling and regulatory functions [42], lymphocyte activation [43] and by blocking the induction of IFN- $\gamma$. Numerous studies have shown the progression of the chlamydial infection cycle to be influenced by IFN- $\gamma$ production by the host. At low IFN- $\gamma$ concentrations acute infections typically occur whereas persistence and clearance of infection occurs at medium and high IFN- $\gamma$ concentrations, respectively $[44,45]$. The ability to block IFN- $\gamma$ production by the host cell may be an important virulence determinant of C. pecorum enabling persistent infection of the host with acute disease symptoms occurring when cytotoxins are overexpressed.

Flanking the cytotoxin genes in C. pecorum are 4 (PV3056/3) or 5 (P787, W73 and E58) phospholipase D (PLD) genes each containing the conserved $\mathrm{HxKx}_{4}$ $\mathrm{Dx}_{6} \mathrm{GSxN}$ (HKD) motif essential for the initiation of phosphodiesterase activity and amino acid motifs that are responsible for catalytic activity. PLD genes identified in the plasticity zone of P787, W73 and E58 share 95-99\% amino acid sequence identity (CPE2_0554, CPE3_0554, G5S_0938; CPE2_0553, CPE3_0553, G5S_0935; CPE2_0551, CPE3_0551, G5S_0931; CPE2_0550, CPE3_0550, G5S_0930) whereas orthologous PLD genes in PV3056/3 are 


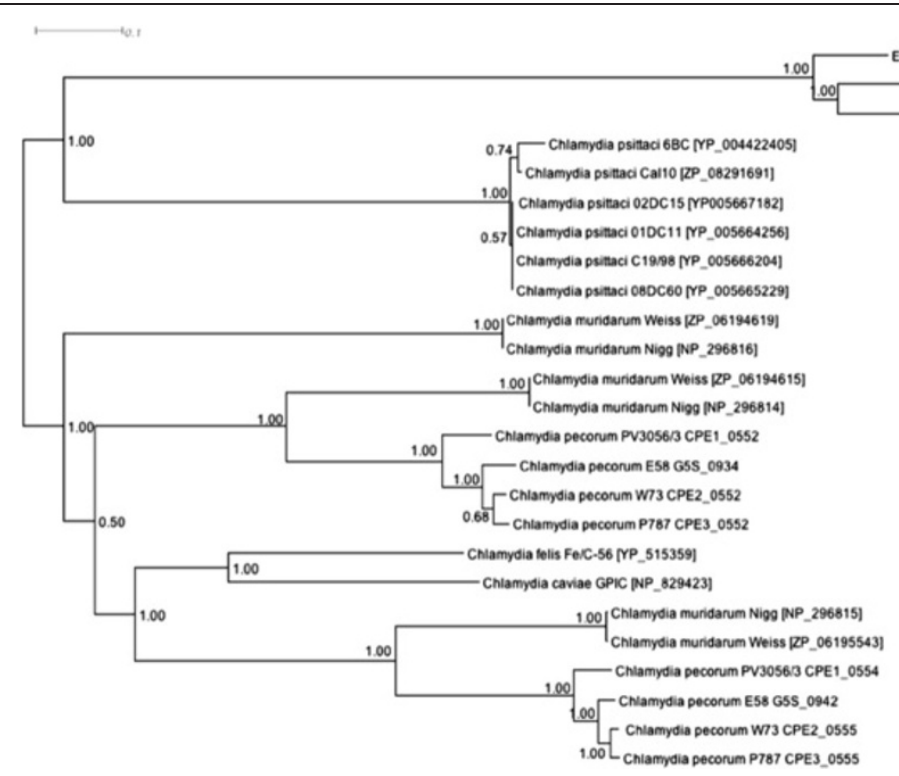

Figure 7 Phylogenetic analysis of chlamydial cytotoxins. Bayesian (MrBayes) phylogenetic tree calculated using a WAG + I $+\mathrm{G}$ substitution model of cytotoxin protein sequences of Chlamydiaceae species. Trees were generated using Markov Chain Monte Carlo settings of 2 runs of 625,000 generations with a burn-in of 125,000 generations with trees sampled every 100 runs. Posterior probabilities are indicated by the number at the node.

more divergent (58-71\% sequence identity) (CPE1_0553, CPE1_0551, CPE1_0550). The remaining PLD gene is almost identical in E58 and W73 (98\% identity, CPE2_0556, G5S_0945) but divergent in the remaining strains (55-79\% identity, CPE1_0555, CPE3_0556). The presence of poly (G) and poly $(\mathrm{C})$ homopolymeric tracts ranging in size from 5-19 nucleotides within the PLD genes and the presence of intact variants in the sequence reads of pseudogenes could indicate that these proteins are subject to phase variation by slip-strand pairing [46]. Whilst the function of PLD in C. pecorum is currently unknown, PLD can perform numerous functions ranging from DNA hydrolysis, to protein-protein interactions with host signalling pathways, to the more classic lipase function. In $C$. trachomatis, PLD genes located in the PZ have been associated with inclusion formation [47], whereas in other bacteria PLD has been identified as an important virulence determinant involved in dissemination, serum resistance and invasion of epithelial cells $[48,49]$.

\section{Conclusions}

The complete genome sequence of C. pecorum P787, W73 and PV3056/3 was determined by Illumina/Solexa and Roche 454 genome sequencing. Despite the differences in the clinical manifestations of infections caused by the strains, comparative analysis revealed a high level of sequence conservation, gene content and order between the genomes. Additional genomic analyses of strains originating from other non-ruminant host species, such as pig and koala, will determine if the high level of sequence similarity is common to all, or just ruminant strains of $C$. pecorum. In agreement with previous studies [20], differences in the number of clustered tandem repeat sequences in ORF663 were observed between strains isolated from diseased (PV3056/3 and P787) or asymptomatic (W73) animals however, no other genetic differences were observed that may account for the different disease manifestations. A number of metabolic traits were identified in $C$. pecorum that may contribute to its ability to evade the host immune system and enable persistent infections to be established in the host. Specifically, this study has particularly highlighted the absence of genes involved in folate biosynthesis and the presence of tryptophan and biotin biosynthesis pathways. The presence of clustered tandem repeats in surface expressed proteins, 15 polymorphic membrane proteins, two cytotoxin genes and multiple phospholipase D genes that are likely to be subject to phase variable expression may play a role in the invasion of host cells and trigger the switching between persistent and acute disease in the host.

\section{Methods}

\section{C. pecorum strain information, propagation and preparation of gDNA}

Three C. pecorum strains originating from different geographical regions and disease manifestations were selected for genome sequencing. Strain P787 was isolated in Scotland, in 1977, from the affected synovial fluid of a sheep with polyarthritis. Strain PV3056/3 was isolated in Italy, in 1991, from a cervical swab of a cow with purulent metritis and has subsequently been shown to induce a 
purulent metritis following inoculation into the uterine body and cervix of cattle [50]. Strain W73 was isolated in Northern Ireland, in 1989, from the faeces of a sheep with an inapparent enteric infection and has subsequently been found to be non-invasive in a mouse model of infection [51].

Strains were propagated in Caco-2 cells grown in RPMI medium supplemented with $5 \%$ FBS and $1 \mu \mathrm{g} / \mathrm{ml}$ cyclohexamide. Genomic DNA from PV3056/3 and P787 was derived from the 7 th tissue culture passage of original strains propagated in fertile hens' eggs. W73 was derived from the 6th tissue culture passage of a strain propagated in fertile hens' eggs, however the passage history prior to this is unknown. Flasks of infected cells were harvested using glass beads followed by centrifugation at 22,000 $\times g$ for 40 mins. Pellets were washed in ice-cold PBS and recentrifuged as before. Pellets were resuspended in $20 \mathrm{mM}$ Tris- $\mathrm{HCl}$ (pH 7.5)/150 mM KCl/1\% sarkosyl and lightly homogenised using a ground glass homogeniser. Homogenised cells were layered onto cushions of $15 \%$ sucrose in $20 \mathrm{mM}$ Tris- $\mathrm{HCl}$ (pH 7.5)/150 mM KCl/1\% sarkosyl and centrifuged at $70,000 \times g$ for $45 \mathrm{~min}$ at $4^{\circ} \mathrm{C}$. Genomic DNA was extracted from pellets using the Wizard DNA extraction kit (Promega).

\section{Genome sequencing}

Genome sequencing was performed by The Gene Pool genomic facility in The University of Edinburgh using Roche 454 GS-FLX and Solexa/Illumina 35-bp paired-end sequencing on standard libraries constructed according to the manufacturers instructions. Reads were assembled using Newbler v2 (Roche) and Velvet v.0.7 [52], combined using minimus 2 and mapped to the reference genome of C. pecorum E58 [24] to generate 13, 10 and 9 contigs for P787, W73 and PV3056/3 respectively. In total, 12,926,259 (PV3056/3), 8,169,259 (W73) and 10,039,539 (P787) reads obtained from Solexa/Illumina sequencing and 95,683 (PV3056/3), 101,405 (W73) and 65,050 (P787) reads from Roche 454 GS-FLX sequencing were obtained. Following quality filtering, sequencing reads were mapped to the reference genome providing approximately $253 \times$ (PV3056/3), 136× (W73) and 59.4× (P787) sequencing coverage. Regions spanning the contig ends were PCRamplified using Phusion High-fidelity DNA polymerase (NEB) and the sequence determined ensuring that each base was covered by sequence in each direction.

\section{Sequence annotation and analysis}

Protein-encoding genes were predicted using Prodigal [53] and open reading frames (ORFs) consisting of fewer than 30 codons or those overlapping larger open reading frames were eliminated. Frameshifts, point mutations and pseudogenes were corrected or confirmed by visual inspection of mapped reads using Tablet [54]. The origin of replication was determined using Ori-finder [55] and the genomes were adjusted so that the first base was upstream of the hemB gene in the oriC region. Ribosomal RNA genes and tRNA genes were identified using RNAmmer and ARAGORN [56,57]. Sequences of experimentally validated small non-coding RNAs (sRNA) from chlamydia were downloaded from BSRD [58] and identified in C. pecorum genomes using blastn. Functional assignments were made based on homology searches using blastp [59] against protein sequences present in the NCBI nr database and the identification of conserved domains using Pfam [60] and InterProScan protein databases [61]. Signal sequences were predicted using the LipoP 1.0 [62]. KEGG orthology assignments were performed using KAAS [63]. Data collation and annotation was performed using Artemis [64].

Comparative analysis were performed using the following genomes: C. pecorum E58 [GenBank: CP002608] [23], C. abortus S26/3 [GenBank: CR848038] [25], C. caviae GPIC [GenBank: AE015925] [26], C. felis Fe/C-56 [GenBank: AP006861] [27], C. psittaci 6BC [GenBank: CP002586] [28], C trachomatis D/UW-3/CX [GenBank: AE001273] [29]), C. pneumoniae AR39 [GenBank: AE0 02161] and C. muridarum Nigg [GenBank: AE002160] [65]. Global genomic comparisons were visualised using ACT [66] with input files generated by the tblastx function in DoubleAct http://www.hpa-bioinfotools.org.uk/ pise/double_act.html\# with a cutoff score of 0 . Comparisons of regions flanking the $\mathrm{PZ}$ were performed using default blastn settings in EasyFig [67]. Orthologous gene sets were identified by OrthoMCL-DB using reciprocal blastp with a cutoff of e-5 and 50\% match [68]. Genome maps were generated using the CGView Server [69].

\section{Phylogenetic analyses}

Reference sequences were obtained from GenBank and aligned with relevant $C$. pecorum CDSs using MUSCLE [70]. Phylogenetic alignments and tree files are available from the Dryad Digital repository http://doi.org/10.5061/ dryad.np597. For ribosomal proteins, 48 individual alignments were concatenated into a single alignment for analysis. For the phylogenetic analysis of cytotoxin genes, GBlocks v 0.91 [71] was used to eliminate regions that could not be unambiguously aligned resulting in 2845 positions being analysed (75\% of the original 3766 positions). Phylogenetic analyses were performed using PhyML (for ribosomal proteins and polymorphic membrane proteins) or MrBayes (for cytotoxins) software [72] launched from the TOPALi v2.5package [73] generated using the JTT $+\mathrm{G}$ (ribosomal proteins), JTT $+\mathrm{I}+\mathrm{G}$ (polymorphic membrane proteins) or $\mathrm{WAG}+\mathrm{I}+\mathrm{G}$ (cytotoxins) substitution model that was determined to be the model of best fit based on the BIC criterion. For MrBayes phylogeny, trees were generated using Markov Chain Monte Carlo (MCMC) settings of 2 runs of 625,000 
generations with a burn-in of 125,000 generations with trees sampled every 100 runs. For PhyML phylogeny, bootstrap analysis was performed based on 100 replicate trees. Phylogenetic network analysis was performed using SplitsTree [74].

\section{Nucleotide sequence accession number}

Genome sequences of C. pecorum strains PV3056/3, W73 and P787 have been deposited in GenBank under the accession numbers CP004033, CP004034, and CP004035, respectively.

\section{Additional files}

\begin{abstract}
Additional file 1: Table S1. Location of small regulatory non-coding RNAs (sRNAs) in C. pecorum genome sequences. Table S2. Identity of pseudogenes in C. pecorum genome sequences. Table S3. Genes involved in folate biosynthesis in Chlamydiaceae species. Table S4. Properties of C. pecorum polymorphic membrane (AT domain-containing) proteins. Table S5. Type III secretion system structural genes and chaperones identified in C. pecorum predicted on the basis of primary sequence similarity (blastp comparison) and domain structure. Table S6. Genetic composition of C. pecorum plasticity zone.
\end{abstract}

Additional file 2: Figure S1. Biotin biosynthesis operon region. Schematic view of the conserved genes dihydropicolinic reductase (dapB) and biotin synthase (bioB) flanking a variable segment positioned upstream of the biotin biosynthesis operon encoding bioBFDA. Dashed lines connect orthologs between the genomes. C. psittaci (locus tags G50_0747-G50_0756) and C. felis (locus tags CF0294-CF0303) have an identical gene arrangement to C. abortus. C. pecorum strains W73, P787 and E58 have an identical arrangement to PV3056/3. Figure S2. Phylogenetic network analysis of Pmp autotransporter domains. Phylogenetic network analysis of Pmp autotransporter domains obtained from aligned AT domain protein sequences using NeighborNet analysis performed through the SplitsTree package [72]. Figure S3. TMH-family proteins. Schematic view showing regions containing predicted Inc-and $\mathrm{TMH}$-family proteins extending between $p m p D$ and $I p \times B$ in members of the family Chlamydiaceae. Pseudogenes in C. pecorum are coloured black. Locus tags are indicated inside each CDS. Dashed lines connect orthologs between genomes. Letters $\mathrm{A}$ and $\mathrm{B}$ indicate the most closely related TMH protein between C. pecorum strains and other chlamydial species. Figure S4. Simple sequence repeats (SSR). (A) Schematic view showing conservation of genes surrounding the SSR region and the positioning of corresponding hypothetical proteins or invasin-like genes in other chlamydial species. Dashed lines connect orthologs between the genomes. The SSR region is indicated by the box. (B) Alignment of nucleotide sequences between the 5S rRNA gene and narF showing the number of repeat sequences (AAAGCACT) in $C$. pecorum. The SSR region is indicated by the box.

\section{Competing interests}

The authors declare that they have no competing interests.

\section{Authors' contributions}

DL conceived of and coordinated the study. BM and SM provided material for genome sequencing. ML, LS, NW and EMC prepared materials for genome sequencing. GM provided reference sequence data used for scaffolding. MS performed finishing sequencing. MS and FAL performed the genome annotations. MS and DL analyzed the results. MS, DL and ML wrote the manuscript. All authors read and approved the final manuscript.

\section{Acknowledgements}

This work as well as MS, NW, LS and EMC was funded by grant no. BB/ E018939/1 from the Biotechnology and Biological Sciences Research Council (BBSRC) and by the Scottish Government Rural and Environment Science and Analytical Services division (RESAS).

\section{Author details}

${ }^{1}$ Moredun Research Institute, Pentlands Science Park, Bush Loan, Edinburgh, Midlothian EH26 OPZ, UK. ${ }^{2}$ School of Veterinary Medicine, College of Agriculture, Food Science and Veterinary Medicine, University College Dublin, Belfield, Dublin 4, Ireland. ${ }^{3}$ Istituto Zooprofilattico Sperimentale della Lombardia e dell'Emilia Romagna "Bruno Ubertini", National Reference Laboratory for Animal Chlamydioses, Sezione Diagnostica di Pavia, Strada Campeggi 61, 27100 Pavia, Italy. ${ }^{4}$ Institute for Genome Sciences, University of Maryland School of Medicine, Baltimore, MD, USA. ${ }^{5}$ Current address: Microbiological Diagnostic Unit, The University of Melbourne, Parkville, Victoria 3010, Australia. ${ }^{6}$ Current address: BigDNA Ltd, Wallace Building, Roslin BioCentre, Roslin, Midlothian EH25 9PP, UK.

Received: 24 September 2013 Accepted: 6 January 2014 Published: 14 January 2014

\section{References}

1. Cavirani S, Cabassi CS, Donofrio G, De laco B, Taddei S, Flammini CF: Association between Chlamydia psittaci seropositivity and abortion in Italian dairy cows. Prev Vet Med 2001, 50:145-151.

2. DeGraves FJ, Gao D, Hehnen HR, Schlapp T, Kaltenboeck B: Quantitative detection of Chlamydia psittaci and C. pecorum by high-sensitivity real-time PCR reveals high prevalence of vaginal infection in cattle. J Clin Microbiol 2003, 41:1726-1729.

3. Jee J, Degraves FJ, Kim T, Kaltenboeck B: High prevalence of natural Chlamydophila species infection in calves. J Clin Microbiol 2004, 42:5664-5672.

4. Lenzko H, Moog U, Henning K, Lederbach R, Diller R, Menge C, Sachse K, Sprague LD: High frequency of chlamydial co-infections in clinically healthy sheep flocks. BMC Vet Res 2011, 7:29.

5. Wilson K, Sammin D, Harmeyer S, Nath M, Livingstone M, Longbottom D: Seroprevalence of chlamydial infection in cattle in Ireland. Vet J 2012, 193:583-585.

6. Yousef Mohamad K, Rodolakis A: Recent advances in the understanding of Chlamydophila pecorum infections, sixteen years after it was named as the fourth species of the Chlamydiaceae family. Vet Res 2010, 41:27.

7. Matsumoto A, Manire GP: Electron microscopic observations on the effects of penicillin on the morphology of Chlamydia psittaci. J Bacteriol 1970, 101:278-285

8. Coles AM, Reynolds DJ, Harper A, Devitt A, Pearce JH: Low-nutrient induction of abnormal chlamydial development: a novel component of chlamydial pathogenesis? FEMS Microbiol Lett 1993, 106:193-200.

9. Raulston JE: Response of Chlamydia trachomatis serovar E to iron restriction in vitro and evidence for iron-regulated chlamydial proteins. Infect Immun 1997, 65:4539-4547.

10. Pantoja LG, Miller RD, Ramirez JA, Molestina RE, Summersgill JT: Characterization of Chlamydia pneumoniae persistence in HEp-2 cells treated with gamma interferon. Infect Immun 2001, 69:7927-7932.

11. Reinhold P, Jaeger J, Liebler-Tenorio E, Berndt A, Bachmann R, Schubert E, Melzer F, Elschner M, Sachse K: Impact of latent infections with Chlamydophila species in young cattle. Vet J 2008, 175:202-211.

12. Jaeger J, Liebler-Tenorio E, Kirschvink N, Sachse K, Reinhold P: A clinically silent respiratory infection with Chlamydophila spp. in calves is associated with airway obstruction and pulmonary inflammation. Vet Res 2007, 38:711-728.

13. Poudel A, Elsasser TH, Rahman KS, Chowdhury EU, Kaltenboeck B: Asymptomatic endemic Chlamydia pecorum infections reduce growth rates in calves by up to 48 percent. PLoS One 2012, 7:e44961.

14. Anderson IE, Baxter SI, Dunbar S, Rae AG, Philips HL, Clarkson MJ, Herring AJ: Analyses of the genomes of chlamydial isolates from ruminants and pigs support the adoption of the new species Chlamydia pecorum. Int I Syst Bacteriol 1996, 46:245-251.

15. Jackson M, Giffard P, Timms P: Outer membrane protein A gene sequencing demonstrates the polyphyletic nature of koala Chlamydia pecorum isolates. Syst Appl Microbiol 1997, 20:187-200.

16. Kaltenboeck B, Kousoulas KG, Storz J: Structures of and allelic diversity and relationships among the major outer membrane protein (ompA) genes of the four chlamydial species. J Bacteriol 1993, 175:487-502.

17. Fukushi H, Hirai K: Genetic diversity of avian and mammalian Chlamydia psittaci strains and relation to host origin. J Bacteriol 1989, 171:2850-2855.

18. Salinas J, Souriau A, De Sa C, Andersen AA, Rodolakis A: Serotype 2-specific antigens from ruminant strains of Chlamydia pecorum detected by 
monoclonal antibodies. Comp Immunol Microbiol Infect Dis 1996, 19:155-161.

19. Liu Z, Rank R, Kaltenboeck B, Magnino S, Dean D, Burall L, Plaut RD, Read TD, Myers G, Bavoil PM: Genomic plasticity of the rrn-nqrF intergenic segment in the Chlamydiaceae. J Bacteriol 2007, 189:2128-2132.

20. Yousef Mohamad K, Roche SM, Myers G, Bavoil PM, Laroucau K, Magnino S, Laurent S, Rasschaert D, Rodolakis A: Preliminary phylogenetic identification of virulent Chlamydophila pecorum strains. Infect Genet Evol 2008, 8:764-771.

21. Yousef Mohamad K, Rekiki A, Myers G, Bavoil PM, Rodolakis A: Identification and characterisation of coding tandem repeat variants in incA gene of Chlamydophila pecorum. Vet Res 2008, 39:56.

22. Jelocnik M, Frentiu FD, Timms P, Polkinghorne A: Multilocus sequence analysis provides insights into molecular epidemiology of Chlamydia pecorum infections in Australian sheep, cattle and koalas. J Clin Microbiol 2013, 51:2625-2632.

23. Mojica S, Huot Creasy H, Daugherty S, Read TD, Kim T, Kaltenboeck B, Bavoil P Myers GS: Genome sequence of the obligate intracellular animal pathogen Chlamydia pecorum E58. J Bacterio/ 2011, 193:3690.

24. Pannekoek Y, Dickx V, Beeckman DSA, Jolley KA, Keijzers WC, Vretou E, Maiden MCJ, Vanrompay D, van der Ende A: Multi locus sequence typing of Chlamydia reveals an association between Chlamydia psittaci genotypes and host species. Plos one 2010, 5:e14179.

25. Thomson NR, Yeats C, Bell K, Holden MT, Bentley SD, Livingstone M, Cerdeño-Tárraga AM, Harris B, Doggett J, Ormond D, Mungall K, Clarke K, Feltwell T, Hance Z, Sanders M, Quail MA, Price C, Barrell BG, Parkhill J, Longbottom D: The Chlamydophila abortus genome sequence reveals an array of variable proteins that contribute to interspecies variation. Genome Res 2005, 15:629-640.

26. Read TD, Myers GSA, Brunham RC, Nelson WC, Paulsen IT, Heidelberg J, Holtzapple E, Khouri H, Federova NB, Carty HA, Umayam LA, Haft DH, Peterson J, Beanan MJ, White O, Salzberg SL, Hsia R-C, McClarty G, Rank RG, Bavoil PM, Fraser CM: Genome sequence of Chlamydophila caviae (Chlamydia psittaci GPIC): examining the role of niche-specific genes in the evolution of the Chlamydiaceae. Nucleic Acids Res 2003, 31:2134-2147.

27. Azuma Y, Hirakawa H, Yamashita A, Cai Y, Rahman MA, Suzuki H, Mitaku S, Toh H, Goto S, Murakami T, Sugi K, Hayashi H, Fukushi H, Hattori M, Kuhara S, Shirai M: Genome sequence of the cat pathogen, Chlamydophila felis. DNA Res 2006, 13:15-23.

28. Grinblat-Huse V, Drabek EF, Creasy HH, Daugherty SC, Jones KM, SantanaCruz I, Tallon LJ, Read TD, Hatch TP, Bavoil P, Myers GS: Genome sequences of the zoonotic pathogens Chlamydia psittaci 6BC and Cal10. J Bacteriol 2011, 193:4039-4040.

29. Stephens RS, Kalman S, Lammel C, Fan J, Marathe R, Aravind L, Mitchell W, Olinger L, Tatusov RL, Zhao Q, Koonin EV, Davis RW: Genome sequence of an obligate intracellular pathogen of humans: Chlamydia trachomatis. Science 1998, 282:754-759.

30. Taylor MW, Feng GS: Relationship between interferon- $\gamma$, indoleamine 2,3dioxygenase, and tryptophan catabolism. FASEB J 1991, 5:2516-2522.

31. Beatty WI, Belanger TA, Desai AA, Morrison RP, Byrne Gl: Tryptophan depletion as a mechanism of gamma interferon-mediated chlamydial persistence. Infect Immun 1994, 62:3705-3711.

32. Eudes A, Erkens GB, Slotboom DJ, Rodionov DA, Naponelli V, Hanson AD: Identification of genes encoding the folate- and thiamine-binding membrane proteins in Firmicutes. J Bacteriol 2008, 190:7591-7594.

33. Myllykallio H, Leduc D, Filee J, Liebl U: Life without dihydrofolate reductase FolA. Trends Microbiol 2003, 11:220-223.

34. Henderson IR, Lam AC: Polymorphic proteins of Chlamydia spp. autotransporters beyond the Proteobacteria. Trends Microbio/ 2001, 9:573-578.

35. Siboo IR, Chambers HF, Sullam PM: Role of SraP, a serine-rich surface protein of Staphylococcus aureus, in binding to human platelets. Infect Immun 2005, 73:2273-2280.

36. Seifert KN, Adderson EE, Whiting AA, Bohnsack JF, Crowley PJ, Brady LJ: A unique serine-rich repeat protein (Srr-2) and novel surface antigen (epsilon) associated with a virulent lineage of serotype III Stretococcus agalactiae. Microbiol 2006, 152:1029-1040.

37. Gravekamp C, Rosner B, Madoff LC: Deletion of repeats in the alpha C protein enhances the pathogenicity of group $B$ streptococci in immune mice. Infect Immun 1998, 66:4347-4354.

38. Rockey DD, Grosenbach D, Hruby DE, Peacock MG, Helnzen RA, Hackstadt T: Chlamydia psittaci IncA is phosphorylated by the host cell and exposed on the cytoplasmic face of the developing inclusion. Mol Microbio/ 1997, 24:217-228.

39. Yousef Mohamad K, Rekiki A, Berri M, Rodolakis A: Recombinant 35-kDa inclusion membrane protein IncA as a candidate antigen for serodiagnosis of Chlamydophila pecorum. Vet Microbiol 2010, 143:424-428.

40. Hackstadt T, Scidmore-Carlson MA, Shaw El, Fisher ER: The Chlamydia trachomatis IncA protein is required for homotypic vesicle fusion. Cell Microbiol 1999, 1:119-130.

41. Geisler WM, Suchland RJ, Rockey DD, Stamm WE: Epidemiology and clinical manifestations of unique Chlamydia trachomatis isolates that occupy nonfusogenic inclusions. J Infect Dis 2001, 184:879-884.

42. Von Eichel-Streiber $C$, Boquet $P$, Sauerborn M, Thelestam M: Large clostridial cytotoxins-a family of glycosyltransferases modifying small GTP-binding proteins. Trends Microbiol 1996, 4:375-382.

43. Klapproth JA, Scaletsky ICA, McNamara BP, Lai L, Malstrom C, James SP, Donnenberg MS: A large toxin from pathogenic Escherichia coli strains that inhibits lymphocyte activation. Infect Immun 2000, 68:2148-2155.

44. Entrican G, Brown J, Graham S: Cytokines and the protective host immune response to Chlamydia psittaci. Comp Immun Microbiol Infect Dis 1998, 21:15-26.

45. Shemer Y, Sarov I: Inhibition of growth of Chlamydia trachomatis by human gamma interferon. Infect Immun 1985, 48:592-596.

46. Viratyosin W, Campbell LA, Kuo CC, Rockey DD: Intrastrain and interstrain genetic variation within a paralogous gene family in Chlamydia pneumoniae. BMC Microbiol 2002, 2:38.

47. Nelson DE, Crane DD, Taylor LD, Dorward DW, Goheen MM, Caldwell HD: Inhibition of chlamydiae by primary alcohols correlates with the strain-specific complement of plasticity zone phospholipase D genes. Infect Immun 2006, 74:73-80.

48. Jacobs AC, Hood I, Boyd KL, Olson PD, Morrison JM, Carson S, Sayood K, Iwen PC, Skaar EP, Dunman PM: Inactivation of phospholipase D diminishes Acinetobacter baumannii pathogenesis. Infect Immun 2010, 78:1952-1962

49. Edwards JL, Entz DD, Apicella MA: Gonococcal phospholipase D modulates the expression and function of complement receptor 3 in primary cervical epithelial cells. Infect Immun 2003, 71:6381-6391.

50. Jones GE, Machell DA, Biolatti B, Appino S: Experimental infections of the genital tract of cattle with Chlamydia psittaci and Chlamydia pecorum. In Proceedings of the Ninth International Symposium on Human Chlamydial Infection. Edited by Stevens RS, Byrne Gl, Christianson G. San Francisco; 1998:446-449.

51. Denamur E, Sayada C, Souriau A, Orfila J, Rodolakis A, Elion J: Restriction pattern of the major outer-membrane protein gene provides evidence for a homogeneous invasive group among ruminant isolates of Chlamydia psittaci. J Gen Microbiol 1991, 137:2525-2530.

52. Zerbino DR, Birney E: Velvet: algorithms for de novo short read assembly using de Bruijn graphs. Genome Res 2008, 18:821-829.

53. Hyatt D, Chen GL, Locascio PF, Land ML, Larimer FW, Hauser LJ: Prodigal: prokaryotic gene recognition and translation initiation site identification. BMC Bioinforma 2010, 11:119.

54. Milne I, Stephen G, Bayer M, Cock PJ, Pritchard L, Cardle L, Shaw P, Marshall $D$ : Using tablet for visual exploration of second-generation sequencing data. Brief Bioinform 2013, 14:193-202

55. Gao F, Zhang CT: Ori-Finder: a web-based system for finding oriCs in unannotated bacterial genomes. BMC Bioinforma 2008, 9:79.

56. Lagesen K, Hallin P, Rødland EA, Staerfeldt HH, Rognes T, Ussery DW: RNAmmer: consistent and rapid annotation of ribosomal RNA genes. Nucleic Acids Res 2007, 35:3100-3108.

57. Laslett D, Canback B: ARAGORN, a program to detect tRNA genes and tmRNA genes in nucleotide sequences. Nucleic Acids Res 2004, 32:11-16.

58. Li L, Huang D, Cheung MK, Nong W, Huang Q, Kwan HS: BSRD: a repository for bacterial small regulatory RNA. Nucleic Acids Res 2013, 41:D233-D238.

59. Atschul SF, Gish W, Miller W, Myers EW, Lipman DJ: Basic local alignment search tool. J Mol Biol 1990, 215:403-410.

60. Finn RD, Tate J, Mistry J, Coggill PC, Sammut JS, Hotz HR, Ceric G, Forslund K, Eddy SR, Sonnhammer EL, Bateman A: The Pfam protein families database. Nucleic Acids Res Database Issue 2008, 36:D281-D288.

61. Zdobnov EM, Apweiler R: InterProScan - an integration platform for the signature-recognition methods in InterPro. Bioinformatics 2001, $17: 847-848$ 
62. Juncker AS, Willenbrock H, von Heijne G, Nielsen H, Brunak S, Krogh A: Prediction of lipoprotein signal peptides in Gram-negative bacteria. Protein Sci 2003, 12:1652-1662.

63. Moriya Y, Itoh M, Okuda S, Yoshizawa A, Kanehisa M: KAAS: an automatic genome annotation and pathway reconstruction server. Nucleic Acids Res 2007, 35:W182-W185.

64. Rutherford K, Parkhill J, Crook J, Horsnell T, Rice P, Rajandream MA, Barrell B: Artemis: sequence visualization and annotation. Bioinformatics 2000, 16:944-945

65. Read TD, Brunham RC, Shen C, Gill SR, Heidelberg JF, White O, Hickey EK, Peterson J, Utterback T, Berry K, Bass S, Linher K, Weidman J, Khouri H, Craven B, Bowman C, Dodson R, Gwinn M, Nelson W, DeBoy R, Kolonay J, McClarty G, Salzberg SL, Eisen J, Fraser CM: Genome sequences of Chlamydia trachomatis MoPn and Chlamydia pneumoniae AR39. Nucleic Acids Res 2000, 15:1397-1406.

66. Carver TJ, Rutherford KM, Berriman M, Rajandream MA, Barrell BG, Parkhill J: ACT: the Artemis comparison tool. Bioinformatics 2005, 21:3422-3423.

67. Sullivan MJ, Petty NK, Beatson SA: Easyfig: a genome comparison visualiser. Bioinformatics 2011, 17:1009-1010.

68. Chen F, Mackey AJ, Stoeckert CJ, Roos DS: OrthoMCL-DB: querying a comprehensive multi-species collection of ortholog groups. Nucleic Acids Res 2006, 34:D363-D368.

69. Grant JR, Stothard P: The CGView server: a comparative genomics tool for circular genomes. Nucleic Acids Res 2008, 36:W181-W184.

70. Edgar RC: MUSCLE: multiple sequence alignment with high accuracy and high throughput. Nucleic Acids Res 2004, 32:1792-1797.

71. Castresana J: Selection of conserved blocks from multiple alignments for their use in phylogenetic analysis. Mol Biol Evol 2000, 17:540-552.

72. Ronquist F, Huelsenbeck JP: MrBayes 3: Bayesian phylogenetic inference under mixed models. Bioinformatics 2003, 19:1572-1574.

73. Milne I, Lindner D, Bayer M, Husmeier D, McGuire G, Marshall DF, Wright F: TOPALi V2: a rich graphical interface for evolutionary analyses of multiple alignments on HPC clusters and multi-core desktops. Bioinformatics 2009, 25:126-127.

74. Huson DH, Bryant D: Application of phylogenetic networks in evolutionary studies. Mol Biol Evol 2006, 23:254-267.

doi:10.1186/1471-2164-15-23

Cite this article as: Sait et al.: Genome sequencing and comparative analysis of three Chlamydia pecorum strains associated with different pathogenic outcomes. BMC Genomics 2014 15:23.

\section{Submit your next manuscript to BioMed Central and take full advantage of:}

- Convenient online submission

- Thorough peer review

- No space constraints or color figure charges

- Immediate publication on acceptance

- Inclusion in PubMed, CAS, Scopus and Google Scholar

- Research which is freely available for redistribution 